Pacific Journal of Mathematics

restro-valutarto volans 


\title{
PSEUDO-VALUATION DOMAINS
}

\author{
John R. Hedstrom and Evan G. Houston
}

\begin{abstract}
A domain $R$ is called a pseudo-valuation domain if, whenever a prime ideal $P$ contains the product $x y$ of two elements of the quotient field of $R$ then $x \in P$ or $y \in P$. It is shown that a pseudo-valuation domain which is not a valuation domain is a quasi-local domain $(R, M)$ such that $V=M^{-1}$ is a valuation overring with maximal ideal $M$. The authors further show that the nonprincipal divisorial ideals of $R$ coincide with the nonzero ideals of $V$. These ideas are then applied to the case of a Noetherian pseudo-valuation domain $R$. Such a domain $R$ is shown to have all its nonzero ideals divisorial if and only if each ideal is two-generated. Examples include valuation rings, certain $D+M$ constructions, and certain rings of algebraic integers.
\end{abstract}

Introduction. The purpose of this paper is to study pseudovaluation domains, a class of rings closely related to valuation rings. We define a pseudo-valuation domain to be a domain $R$ in which every prime ideal $P$ has the property that whenever a product of two elements of the quotient field of $R$ lies in $P$ then one of the given elements is in $P$. One shows easily that valuation rings are pseudo-valuation domains (Prop. 2.1). In the first section of the paper, several characterizations of pseudo-valuation domains are given. For example, a quasi-local domain $(R, M)$ is a pseudo-valuation domain if and only if $x^{-1} M \subset M$ whenever $x$ is an element of the quotient field of $R, x \notin R$ (Th. 1.4).

The name "pseudo-valuation domain" is justified in the second section, first by showing that these rings share many properties with valuation rings. More important is the characterization of a pseudovaluation domain which is not a valuation domain as a quasi-local domain $(R, M)$ with the property that $V=M^{-1}$ is a valuation overring with maximal ideal $M$. The second section is concluded with a study of the relationship between the ideals of $R$ and the ideals of $V$; for example, the set of nonzero ideals of $V$ and the set of nonprincipal, divisorial ideals of $R$ are shown to be one and the same (Cor. 2.15).

In the final section, the authors study Noetherian pseudo-valuation domains. Such rings have Krull dimension $\leqq 1$. Also, a Noetherian pseudo-valuation domain has the 2-generator property if and only if every nonzero ideal is divisorial (Th. 3.5).

Besides valuation rings, two other classes of examples of pseudo- 
valuation domains are given. The first (Ex. 2.1) is obtained by taking a valuation ring of the form $V=K+M, K$ a field, and taking $R$ to be a subring of the form $F+M, F$ a proper subfield of $K$. A second class of (Noetherian) pseudo-valuation domains is provided by localizing certain rings of algebraic integers (Ex. 3.6).

\section{Definitions and properties.}

Definition. Let $R$ be a domain with quotient field $K$. A prime ideal $P$ of $R$ is called strongly prime if $x, y \in K$ and $x y \in P$ imply that $x \in P$ or $y \in P$.

Definition. $A$ domain $R$ is called a pseudo-valuation domain if every prime ideal of $R$ is strongly prime.

Proposition 1.1. Every valuation domain is a pseudo-valuation domain.

Proof. Let $V$ be a valuation domain, and let $P$ be a prime ideal in $V$. Suppose $x y \in P$ where $x, y \in K$, the quotient field of $V$. If both $x$ and $y$ are in $V$, we are done. Suppose that $x \notin V$. Since $V$ is a valuation domain, we have $x^{-1} \in V$. Hence $y=x y \cdot x^{-1} \in P$, as desired.

As we shall see in the next section, the converse of the above proposition is false. We turn now to some simple properties and characterizations of pseudo-valuation domains.

Proposition 1.2. Let $P$ be a prime ideal of a domain $R$ with quotient field $K$. Then $P$ is strongly prime if and only if $x^{-1} P \subset P$ whenever $x \in K-R$.

Proof. Assume that $P$ is strongly prime. If $x \in K-R$ and $p \in P$ then $p=p x^{-1} \cdot x \in P$, whence $p x^{-1} \in P$ or $x \in P$. Since $x \notin R$ we must have $p x^{-1} \in P$. Thus $x^{-1} P \subset P$.

Conversely, assume $x^{-1} P \subset P$ whenever $x \in K-R$, and let $a b \in$ $P$. If $a, b \in R$ there is nothing to prove. Hence we may assume $a \notin R$ so that $a^{-1} P \subset P$ and $b=a^{-1} \cdot a b \in P$. This completes the proof.

COROLlARY 1.3. In a pseudo-valuation domain $R$, the prime ideals are linearly ordered. In particular $R$ is quasi-local.

Proof. Let $P$ and $Q$ be prime ideals, and suppose $a \in P-$ 
$Q$. Then for each $b \in Q$ we have $a / b \notin R$. Hence $(b / a) P \subset P$ by the proposition. Thus $b=b / a \cdot a \in P$ and we have $Q \subset P$.

THEOREM 1.4. Let $(R, M)$ be a quasi-local domain. The following statements are equivalent.

(1) $R$ is a pseudo-valuation domain.

(2) For each pair I, $J$ of ideals of $R$, either $I \subset J$ or $M J \subset M I$.

(3) For each pair I, $J$ of ideals of $R$, either $I \subset J$ or $M J \subset I$.

(4) $M$ is strongly prime.

Proof. (1) $\Rightarrow(2)$. Assume $I \not \subset J$ and pick $a \in I-J$. For each $b \in J$ we have $a / b \notin R$, so that $(b / a) M \subset M$ and $M b \subset M a \subset M I$. It follows that $M J \subset M I$.

(2) $\Rightarrow(3)$. This requires no comment.

(3) $\Rightarrow$ (4). Let $a, b \in R$ with $a / b \notin R$. We shall show that $(b / a) M \subset M$; by Proposition 1.2 this will suffice. Since $a / b \notin R$ we have $(a) \not \subset(b)$ whence $M b \subset(a)$ and $M b / a \subset R$. If $M b / a=R$ then $M=R a / b$ and $a / b \in R$, a contradiction. Hence $M b / a \subset M$, as was to be shown.

(4) $\Rightarrow(1)$. Let $x$ be an element of the quotient field of $R, x \notin R$, and let $P$ be a prime ideal. Again, by Proposition 1.2, it is enough to show that $x^{-1} P \subset P$. Accordingly, let $p \in P$ and note that since $P \subset M$, we have $x^{-1} p \in M$. Hence $x^{-1} p \cdot x^{-1} \in M$, whence $\left(x^{-1} p\right)^{2}=$ $x^{-1} p x^{-1} \cdot p \in P$. Since $P$ is prime and $x^{-1} p \in R$, we therefore have $x^{-1} p \in P$.

In the following theorem we characterize pseudo-valuation domains without making the quasi-local assumption.

THEOREM 1.5. Let $R$ be a domain with quotient field $K$. The following statements are equivalent.

(1) $R$ is a pseudo-valuation domain.

(2) For each $x \in K-R$ and for each nonunit a of $R$, we have $(x+a) R=x R$.

(3) For each $x \in K-R$ and for each nonunit $a$ of $R$, we have $x^{-1} a \in R$.

Proof. (1) $\Rightarrow$ (2) Let $x \in K-R$ and let $a$ be a nonunit of $R$. Then $a \in P$ for some prime ideal $P$, so that $x^{-1} a \in x^{-1} P \subset P \subset$ $R$. Hence $(x+a) / x=1+a / x \in R$ and $(x+a) R \subset x R$. On the other hand, $x+a \notin R$ so that $(x+a)^{-1} P \subset P$ and $a /(x+a) \in R$. Since $x /(x+a)=1-a /(x+a)$, we have $x /(x+a) \in R$ and $x R \subset(x+a) R$.

(2) $\Rightarrow$ (3). By (2) $(x+a) / x=1+a / x \in R$, whence $x^{-1} a \in R$ also.

(3) $\Rightarrow$ (1). Let $P$ be prime and take $a b \in P$ with $a, b \in K$. We 
may assume $b \notin R$. By hypothesis since $a b$ is a nonunit of $R, a=$ $b^{-1} \cdot a b \in R$. We claim that $a$ is a nonunit; otherwise $b=a^{-1} \cdot a b \in P$, a contradiction. We apply the hypothesis again to get $b^{-1} a \in R$. Thus $a^{2}=b^{-1} a \cdot a b \in P$ and $a \in P$, as desired.

We close this section with a brief study of overrings of pseudovaluation domains. (By an overring of a domain $R$, we mean a domain between $R$ and its quotient field.)

Lemma 1.6. Let $R$ be a pseudo-valuation domain and let $T$ be an overring. If $Q$ is prime in $T$, then every prime ideal of $R$ contained in $Q \cap R$ is also a prime ideal of $T$.

Proof. Let $P$ be prime in $R$ with $P \subset Q \cap R$. To show that $P$ is an ideal of $T$, it suffices to show $t p \in P$ for all $t \in T, p \in P$. Now $p=t p \cdot t^{-1} \in P \Rightarrow t p \in P$ or $t^{-1} \in P$. However, if $t^{-1} \in P \subset Q \cap R$, we have that $t^{-1} \in Q$. This implies that $t^{-1}$ is a nonunit of $T$, contradicting that $t \in T$. Thus $t p \in P$ and $P$ is indeed an ideal of $T$. That $P$ is a prime ideal of $T$ follows easily from the fact that $P$ is strongly prime in $R$.

THEOREM 1.7. Let $R$ be a pseudo-valuation domain with overring $T$. If the pair $R \subset T$ satisfies incomparability, then $T$ is also a pseudovaluation domain, and every prime ideal of $T$ is a prime of $R$.

Proof. Let $Q$ be a prime ideal of $T$. We claim that $Q$ is also prime in $R$. Clearly $Q \cap R$ is prime in $R$, whence $Q \cap R$ is prime in $T$ by the lemma. Thus $Q \cap R \subset Q$ are primes of $T$ lying over $Q \cap R$ in $R$. Since incomparability holds, we must have $Q=Q \cap R$, so that $Q$ is a prime of $R$. Since $R$ and $T$ have the same quotient field and $Q$ is strongly prime in $R$, it follows easily that $Q$ is strongly prime in $T$. Thus $T$ is a pseudo-valuation domain.

II. Valuation overrings. We begin this section with an example which anticipates most of the results in the section.

EXAMPLE 2.1. Let $V$ be a valuation domain of the form $K+M$, where $K$ is a field and $M$ is the maximal ideal of $V$. If $F$ is a proper subfield of $K$, then $R=F+M$ is a pseudo-valuation domain which is not a valuation domain. To see this, note that by $[3$, Theorem A, p. 560] $R$ and $V$ have the same quotient field $L$ and that $M$ is the maximal ideal of $R$. Therefore, since valuation domains are pseudo-valuation domains, we see that $M$ is strongly prime in $V$. It follows from the fact that $R$ and $V$ have the same quotient field that $M$ is strongly prime in $R$. Thus by 
Theorem $1.4 R$ is a pseudo-valuation domain. Note that $R$ is not a valuation ring, again by $[3$, Theorem A, p. 560].

Proposition 2.2. If a GCD domain $R$ is also a pseudo-valuation domain, then $R$ is a valuation domain.

Proof. By Theorem 1.3 the primes of $R$ are linearly ordered. Thus the result follows from [7, Theorem 1].

REMARK 2.3. It is not enough in the above proposition to take $R$ to be an integrally closed pseudo-valuation domain, for if in Example 2.1 we take $F$ to be algebraically closed in $K$, then we have by [3, Theorem A, p. 560] that $R$ is integrally closed.

As the following results show, pseudo-valuation domains enjoy many of the same properties that valuation domains do.

Proposition 2.4. If $I$ is an ideal in a pseudo-valuation domain, then $P=\cap\left\{I^{k}: k=1,2, \cdots\right\}$ is a prime ideal.

Proof. Let $x y \in P$ with $x \notin P$. Since $x \notin P$ we have that $x \notin I^{n}$ for some $n>0$. Thus by Theorem $1.4 I^{2 n} \subset(x)$. Hence for each positive integer $k$, we have $(x y) \subset P \subset I^{2 n+k}=I^{2 n} \cdot I^{k} \subset x I^{k}$, whence $y \in I^{k}$. Therefore $y \in P$ and $P$ is prime.

Corollary 2.5. Let $I, J$ be ideals in a pseudo-valuation domain $R$. If $I \varsubsetneqq \sqrt{J}$ then $J$ contains some power of $I$.

Proof. Suppose $I^{k} \not \subset J$ for all $k>0$. Then by Theorem 1.4 we have $J^{2} \subset I^{k}$ for all $k$ so that $J^{2} \subset \cap\left\{I^{k}: k=1,2, \cdots\right\}=P$, a prime ideal. Hence $J \subset P \subset I$ and $\sqrt{ } J \subset P \subset I$, a contradiction.

Proposition 2.6. Let $R$ be a pseudo-valuation domain with maximal ideal $M$. If $P$ is a nonmaximal prime ideal of $R$, then $R_{P}$ is a valuation domain.

Proof. Let $K$ denote the quotient field of $R$, and let $x \in K$. If $x \in R$ then $x \in R_{P}$. If $x \notin R$ then since $R$ is a pseudo-valuation domain $x^{-1} M \subset M$. Choose $m \in M-P$. Then $x^{-1}=x^{-1} m / m \in R_{P}$.

We now characterize pseudo-valuation domains in terms of valuation overrings.

THEOREM 2.7. The following statements are equivalent for a quasilocal domain $(R, M)$. 
(1) $R$ is a pseudo-valuation domain.

(2) $R$ has a (unique) valuation overring $V$ with maximal ideal $M$.

(3) There exists a valuation overring $V$ in which every prime ideal of $R$ is also a prime ideal of $V$.

Proof. (1) $\Rightarrow(2) \quad$ By [5, Theorem 56] there is a valuation overring $(W, N)$ with $N \cap R=M$. By Lemma 1.6 M is a prime ideal of $W$. Put $V=W_{M}$, then $V$ is a valuation overring with maximal ideal $M_{M}$. Since $M$ is strongly prime, it follows easily that $M=M_{M}$. The uniqueness of $V$ follows from the fact that valuation overrings of $R$ are determined by their maximal ideals [3, Theorem 14.6].

(2) $\Rightarrow(3)$. Let $P$ be prime in $R, p \in P$, and $v \in V$. Then $p \in M$ so that $v p \in M$. Thus $v^{2} p \in M$, whence $(v p)^{2} \in P$. Hence $v p \in P$ and $P$ is an ideal of $V$. Now let $x y \in P$ with $x, y \in V$. If both $x$ and $y$ are in $R$ then $x \in P$ or $y \in P$. Thus assume $x \notin R$ so that $x \notin M$ and $x^{-1} \in V$. Thus, since $P$ is an ideal of $V$, we have $y=$ $x^{-1} \cdot x y \in P$. Hence $P$ is a prime ideal of $V$.

(3) $\Rightarrow(1)$. Let $V$ be the given valuation overring. Then since every prime ideal $P$ of $R$ is also prime in $V$, and since $V$ is a pseudo-valuation domain, $P$ is strongly prime. Thus $R$ is a pseudovaluation domain.

In Theorem 2.10 we shall give more information about the valuation overring in the above theorem. We have need of the following:

Proposition 2.8. Let $(R, M)$ be a pseudo-valuation ring which is not a valuation ring, and let $(V, M)$ be the valuation overring (of Theorem 2.7). If $I$ is a nonzero principal ideal of $R$, then $I$ is not an ideal of $V$.

Proof. Suppose $I=R a$ is a nonzero ideal of $V$. Then $I=V I=$ $V R a=V a$. Choose $v \in V-R$. Then $v a \in I$ so that $v a=r a$ with $r \in R$ and $v=r \in R$, a contradiction.

COROllary 2.9. If a pseudo-valuation domain $R$ has a nonzero principal prime ideal, then $R$ is a valuation domain.

Proof. Assume that $R$ is not a valuation domain. Let $V \neq R$ be a valuation overring with the same maximal ideal. If $P$ is a nonzero principal prime ideal of $R$ then $P$ is not an ideal of $V$ by Proposition 2.8. This contradicts Lemma 1.6.

We now show that the valuation overring of Theorem 2.7 (2) is simply $M^{-1}$.

THEOREM 2.10. Let $(R, M)$ be a quasi-local domain which is not a 
valuation domain. Then $R$ is a pseudo-valuation domain if and only if $V=M^{-1}$ is a valuation overring with maximal ideal $M$.

Proof. Assume that $R$ is a pseudo-valuation domain. Let $x \in$ $V=M^{-1}$. We claim that $x M \subset M$. Otherwise $x M=R$, whence $M=$ $R x^{-1}$ is principal and $R$ is a valuation domain by Corollary 2.9. Since $R$ was assumed not valuation, our claim is verified. To show that $V$ is an overring, it suffices to show that $x y \in V$ whenever $x, y \in V$. This follows from our claim since $x, y \in V$ implies $x y M \subset x M \subset M \subset R$ so that $x y \in M^{-1}=V$. To see that $V$ is a valuation domain, let $z$ be an element of the quotient field. If $z \in R$ then $z \in V$. Otherwise, $z^{-1} M \subset M$, whence $z^{-1} \in M^{-1}=V$. That $M$ is an ideal of $V$ also follows from $x M \subset M$ whenever $x \in V$. To see that $M$ is the maximal ideal of $V$, let $x$ be a nonunit of $V$. If $x \notin M$ then $x \notin R$, whence $x^{-1} M \subset M$ and $x^{-1} \in V$, a contradiction. Thus $M$ is the maximal ideal of $V$.

Conversely, assume that $V=M^{-1}$ is a valuation ring with maximal ideal $M$. Then $R$ is a pseudo-valuation domain by Theorem 2.7.

Throughout the rest of this section, $(R, M)$ will denote a pseudovaluation domain which is not a valuation ring, and $V=M^{-1}$ will denote the valuation overring with the same maximal ideal. As we have seen (Theorem 2.7), every prime ideal of $R$ is also a prime ideal of $V$. Conversely, since every ideal of $V$ is contained in $M$, it is clear that every ideal of $V$ is an ideal of $R$. Thus $R$ and $V$ have the same set of prime ideals. As Proposition 2.8 shows, however, if $A$ is a nonzero ideal of $V$ then $A$ is not a principal ideal of $R$; hence there are ideals of $R$ which are not ideals of $V$. We shall now study further the relationship between ideals of $R$ and ideals of $V$. This study is motivated by Bastida and Gilmer's investigation of divisorial ideals in rings of the form $D+M[1, \S 4]$. In particular, compare [1, Theorem 4.1] with Lemma 2.12 and [1, Theorem 4.3 (1)] with Theorem 2.13.

Proposition 2.11. If $A$ is an ideal of $R$, then either $A$ is an ideal of $V$ or $A V$ is a principal ideal of $V$.

Proof. Assume that $A$ is not an ideal of $V$, and choose $x \in A V-$ $A$. We shall show that $A V=x V$. Suppose, on the contrary, that $y \in A V-x V$. Then $y / x \notin V$, so that $x / y \in M$ and $x=$ $x / y \cdot y \in M(A V)=M A \subset A$, a contradiction. Thus $A V=x V$ is a principal ideal of $V$.

To complete our discussion of ideals we have need of the $v$ operation, a discussion of which may be found in [1, p. 87]. To simplify our notation, we shall use " $v$ " for the $v$-operation on $R$ and " $w$ " for the $v$-operation on $V$. Recall that an ideal $A$ is called divisorial $\Leftrightarrow A$ is a 
$v$-ideal $\Leftrightarrow A=A_{v}=\left(A^{-1}\right)^{-1}=$ the intersection of principal fractional ideals containing $A$.

\section{LeMma 2.12. $M$ is divisorial.}

Proof. Otherwise $M^{-1}=R$, contradicting that $M^{-1}$ is a valuation overring.

TheOREM 2.13. If $A$ is a nonzero ideal of $V$, then $A$ is a divisorial ideal of $R$.

Proof. We have already noted that $A$ is an ideal of $R$. Assume that $A$ is not divisorial in $R$, and pick $x \in A_{v}-A$. We assert that $M x=M A$. Since $R x \not \subset A$ we have $M A \subset M x$ by Theorem 1.4. Furthermore, if $M x \not \subset M A$ then $A \subset R x$, also by Theorem 1.4. Hence if $a \in A$ then $a=r x$, whence $r \in M$ since $x \notin A$. Thus $a \in M x$ and $A \subset M x$. This implies that $R x \subset A_{v} \subset(M x)_{v}=M_{v} x=M x$, the last equality following from the lemma. We have arrived at the absurdity that $R x \subset M x$; therefore, $M x=M A$ as asserted.

Now in $V$ either $M_{w}=V$ or $M$ is principal [1, Lemma 4.2]. In either case $M_{w}$ is principal. Thus $M_{w} x=(M x)_{w}=(M A)_{w}=\left(M_{w} A_{w}\right)_{w}=$ $M_{w} A_{w}$, the last equality following from the fact that $M_{w}$ is principal. Again, since $M_{w}$ is principal, we cancel $M_{w}$ from the equation $M_{w} x=M_{w} A_{w}$, yielding $V x=A_{w}$. If $A_{w}=A$ then $x \in A_{w}=A$, a contradiction. Thus $A$ is not divisorial in $V$, whence by [1, Lemma 4.2], $A=b M$ for some $b \in K$, the quotient field of $V$. But then $A_{v}=$ $(b M)_{v}=b M_{v}=b M=A$, and the theorem is established.

Proposition 2.14. If $A$ is an ideal of $R$, then either $A$ is principal in $R$ or $A_{v}=A V$.

Proof. Suppose $A$ is not principal. Since $A V$ is an ideal of $V, A V$ is a divisorial ideal of $R$ by the preceding theorem. Thus since $A \subset A V$ we have $A_{v} \subset(A V)_{v}=A V$. We must prove that $A V \subset A_{v}$; thus if $x \in A^{-1}$ we must show $A V x \subset R$. But $x \in A^{-1}$ implies that $x A \subset R$ whence $x A \subset M$ since $A$ is not principal. Hence $V x A \subset V M=M \subset R$, as desired.

Corollary 2.15. $A$ is a divisorial ideal of $R$ if and only if $A$ is a nonzero principal ideal of $R$ or $A$ is a nonzero ideal of $V$.

Proof. If $A$ is a nonzero principal ideal of $R$, then $A$ is clearly divisorial. If $A$ is a nonzero ideal of $V$, then $A$ is divisorial in $R$ by Theorem 2.13. 
Conversely, assume that $A$ is a divisorial ideal of $R$. If $A$ is not principal, then $A_{v}=A V$ by the preceding result. Hence $A=A_{v}=A V$ is an ideal of $V$.

REMARK. A summary of the results in $2.7-2.15$ is in order. Let $(R, M)$ be a pseudo-valuation domain which is not a valuation ring. Then $V\left(=M^{-1}\right)$ is a valuation overring whose prime ideals coincide with those of $R$ (Theorem 2.7 and 2.10). Recall that each nonzero ideal of $V\left(=M^{-1}\right)$ is a nonprincipal ideal of $R$ (Proposition 2.8). On the other hand, a nonprincipal ideal $I$ of $R$ is an ideal of $V \Leftrightarrow I$ is divisorial in $R$ (Corollary 2.15). Thus the nonprincipal divisorial ideals of $R$ coincide with the nonzero ideals of $V$.

\section{Noetherian pseudo-valuation domains.}

THEOREM 3.1. Let $R$ be a Noetherian domain with quotient field $K$ and integral closure $R^{\prime}$. Then $R$ is a pseudo-valuation domain if and only if $x^{-1} \in R^{\prime}$ whenever $x \in K-R$.

Proof. Assume that $R$ is a pseudo-valuation domain with maximal ideal $M$. If $x \in K-R$ then $x^{-1} M \subset M$. Since $M$ is finitely generated, we have $x^{-1} \in R^{\prime}$ by [5, Theorem 12].

Conversely, assume $x \in K-R$ and let $P$ be prime in $R$. We must show $x^{-1} P \subset P$.

Let $P^{\prime}$ be a prime ideal of $R^{\prime}$ such that $P^{\prime} \cap R=P[5$, Theorem 44]. Since $x^{-1} \in R^{\prime}, x^{-1} P \subset x^{-1} P^{\prime} \subset P^{\prime}$. We claim $x^{-1} P \subset R$, in which case $x^{-1} P \subset P^{\prime} \cap R=P$, and we are done. To prove the claim, suppose there exists $p \in P$ with $x^{-1} p \notin R$. Then $x p^{-1} \in R^{\prime}$ by hypothesis, whence $1=x p^{-1} \cdot x^{-1} p \in P^{\prime}$, a contradiction.

Proposition 3.2. If $R$ is a Noetherian pseudo-valuation domain, then $R$ has Krull dimension $\leqq 1$.

Proof. This follows from [5, Theorem 144] and the fact that the primes of $R$ are linearly ordered (Corollary 1.3).

Corollary 3.3. If $R$ is a Noetherian pseudo-valuation domain, then every overring of $R$ is a pseudo-valuation domain.

Proof. By the Krull-Akizuki Theorem [5, Theorem 93], every overring $T$ has Krull dimension $\leqq 1$ (and is Noetherian). Hence the pair $R \subset T$ satisfies incomparability, and $T$ is a pseudo-valuation domain by Theorem 1.7 . 
COROllary 3.4. If $R$ is a Noetherian pseudo-valuation domain, then the integral closure $R^{\prime}$ of $R$ is a discrete rank one valuation ring.

Proof. We noted in the proof of Corollary 3.3 that $R^{\prime}$ is a pseudo-valuation ring, hence $R^{\prime}$ is local of Krull dimension one and integrally closed. Thus $R^{\prime}$ is a discrete rank one valuation ring.

REMARK. A Noetherian pseudo-valuation domain which is a $G C D$ domain is a discrete rank one valuation ring by Proposition 2.2.

In Theorem 3.5 we prove that each nonzero ideal of a Noetherian pseudo-valuation domain is divisorial if and only if every ideal of $R$ requires at most two generators. The result is a consequence of Matlis [6, Theorems 40 and 57]. We include our direct proof due to the considerable simplification of the Matlis results in the case where $R$ is a pseudo-valuation domain. It should be noted that the conditions on $R$ in Theorem 3.5 do not imply that $R$ is a pseudo-valuation domain, as one can show using the example in [2, Exercise 1, p. 81].

TheOREM 3.5. Let $(R, M)$ be a Noetherian pseudo-valuation domain with $V=M^{-1}(\neq R)$ its valuation overring. Then the following statements are equivalent.

(1) Each nonzero ideal of $R$ is divisorial.

(2) Each ideal of $R$ may be generated by two elements.

(3) $M$ may be generated by two elements.

(4) $V$ is a two-generated $R$-module.

(5) Each nonprincipal ideal of $R$ is an ideal of $V$.

Proof. (1) $\Leftrightarrow$ (5) This is a restatement of Corollary 2.15.

(1) $\Rightarrow$ (2) By [4, Lemma 2.2], $V=R+R x$ with $x \in V-R$. Let $I$ be a nonprincipal ideal of $R$. By (5) $I=I V=k V$ for some $k \in I$ since $V$ is a discrete rank one valuation ring. Hence $I=k V=k(R+R x)=$ $R k+R k x$, and $I$ is two-generated.

(2) $\Rightarrow$ (3). This is trivial.

(3) $\Rightarrow(4)$. Let $M=(a, b)$. Then in $V, M$ is generated by one of $a$ and $b$, say $M=a V$. Then $V=1 / a M=1 / a(R a+R b)=R+R b / a$, and $V$ is two-generated.

(4) $\Rightarrow$ (5). Write $V=R x+R y$. We first reduce to the case $y=$ 1. To this end pick $r, s \in R$ with $1=r x+s y$. Then either $r$ or $s$, say $s$, is a unit, and $y=s^{-1}-s^{-1} r x \in R+R x$. Thus $V=R+R x$. Now let $I$ be a nonprincipal ideal of $R$. Then $I V=k V$ for some $k \in I$, and, since $I$ is not principal in $R$, we may pick $i \in I-k R$. Now $i=k v=k(a+b x)$ for some $a, b \in R, v \in V$. If $b \in M$, then $b x \in M$ whence $a+b x \in R$ and $i \in k R$, a contradiction. Hence $b$ is a unit of $R$, and we have 
$k x=b^{-1} i-b^{-1} k a \in I$. Thus $I V=k V=k K+k x R \subset I$, proving (5).

We close this section with an example of a Noetherian pseudovaluation domain which is not a valuation ring. The example given is easily seen to satisfy the equivalent conditions of Theorem 3.5.

EXAMPLE 3.6. Let $m$ denote a square-free positive integer, $m \equiv 5$ $(\bmod 8)$. Let $Z$ denote the ring of integers and set $D=Z[\sqrt{m}]$. Since $m \equiv 1(\bmod 4), D$ does not contain the algebraic integers of the form $(a+b \sqrt{m}) / 2$, where $a$ and $b$ are odd integers. Thus, $D$ is not integrally closed [8, Theorem 6.6]. It is routine to check that $(2,1+\sqrt{m})=N$ is a maximal ideal of $D$. The desired example is $R=D_{N}$, which has $K=Q[\sqrt{m}]$ as its quotient field. $R$ is not a valuation ring since neither $(1+\sqrt{m}) / 2$ nor its inverse lies in $R$.

To show that $R$ is a pseudo-valuation ring we apply Theorem 3.1 to the integral closure $R^{\prime}$ of $R$. Since $R^{\prime}=\left(D_{N}\right)^{\prime}=\left(D^{\prime}\right)_{S}$, where $S=$ $D-N$ and (') denotes integral closure, we must show $x \in K-R$ implies $1 / x \in\left(D^{\prime}\right)_{s}$. Now $\quad x=(a+b \sqrt{m}) / c \quad$ where $a, b, c \in Z$ and $\operatorname{gcd}(a, b, c)=1$. Since $x \notin R, c \in N \cap Z=2 Z$ so 2 divides $c$. But then $a$ or $b$ must be odd since $\operatorname{gcd}(a, b, c)=1$. Now $x^{-1}=c(a-b \sqrt{m})$. $\left(a^{2}-b^{2} m\right)^{-1}$. If $a^{2}-b^{2} m \notin S$ then $a^{2}-b^{2} m \in N \cap Z=2 Z$, but $m \equiv 1$ $(\bmod 4)$; so $a$ and $b$ are both odd integers. It follows that $a^{2}-b^{2} m \equiv 0$ $(\bmod 4)$, but $a^{2}-b^{2} m \equiv 1-m \equiv 4(\bmod 8)$. Thus $a^{2}-b^{2} m=4 t$ with $t$ an odd integer, and so $x^{-1}=(c / 2((a-b \sqrt{m}) / 2)) / t \in D_{s}^{\prime}=R$ because with $a, b$ odd integers we have $(a-b \sqrt{m}) / 2$ an algebraic integer, hence an element of $D^{\prime}$.

We are grateful to the referee for his many helpful suggestions.

\section{REFERENCES}

1. E. Bastida and R. Gilmer, Overrings and divisorial ideals of rings of the form $D+M$, Michigan Math. J., 20 (1973), 79-95.

2. N. Bourbaki, Elements de Mathemathique, Algebra Commutative, XXXI, Hermann, Paris, 1965.

3. R. Gilmer, Multiplicative Ideal Theory, Queens Papers on Pure and Applied Mathematics, No.

12. Queens University Press, Kingston, Ontario, 1968.

4. W. Heinzer, Integral domains in which each non-zero ideal is divisorial, Mathematika, 15 (1968), 164-170.

5. I. Kaplansky, Commutative Rings, Allyn and Bacon, Boston, 1970.

6. E. Matlis, Torsion-free modules, Chicago Lectures in Mathematics, The University of Chicago Press, Chicago, 1972.

7. S. McAdam, Two Conductor Theorems, J. Algebra, 23 (1972), 239-240.

8. H. Pollard, The Theory of Algebraic Numbers, The Carus Mathematical Monographs, No. 9, M.A.A., John Wiley and Sons, New York, 1961.

Received October 11, 1976 and in revised form March 18, 1977. 



\section{PACIFIC JOURNAL OF MATHEMATICS EDITORS}

ICHARD ARENS (Managing Editor)

niversity of California

os Angeles, CA 90024

\section{. A. BEAUmont}

niversity of Washington

sattle, WA 98105

. C. MOORE

niversity of California

erkeley, CA 94720
J. DUGUNDJI

Department of Mathematics

University of Southern California

Los Angeles, CA 90007

R. FINN AND J. MiLgRAM

Stanford University

Stanford, CA 94305

\section{ASSOCIATE EDITORS}
F. BECKENBACH
B. H. NEUMANN
F. WOLF
K. YosHidA

\section{SUPPORTING INSTITUTIONS}

NIVERSITY OF BRITISH COLUMBIA

UNIVERSITY OF SOUTHERN CALIFORNIA

ALIFORNIA INSTITUTE OF TECHNOLOGY STANFORD UNIVERSITY

NIVERSITY OF CALIFORNIA

ONTANA STATE UNIVERSITY

UNIVERSITY OF HAWAII

NIVERSITY OF NEVADA

UNIVERSITY OF TOKYO

EW MEXICO STATE UNIVERSITY

UNIVERSITY OF UTAH

REGON STATE UNIVERSITY

NIVERSITY OF OREGON

WASHINGTON STATE UNIVERSITY

UNIVERSITY OF WASHINGTON

SAKA UNIVERSITY

AMERICAN MATHEMATICAL SOCIETY

The Supporting Institutions listed above contribute to the cost of publication of this Journal, but they a t owners or publishers and have no responsibility for its contents or policies.

Mathematical papers intended for publication in the Pacific Journal of Mathematics should be in typ Irm or offset-reproduced (not dittoed), double spaced with large margins. Underline Greek letters in re ierman in green, and script in blue. The first paragraph or two must be capable of being used separately as 'nopsis of the entire paper. Items of the bibliography should not be cited there unless absolutely necessary, hich case they must be identified by author and Journal, rather than by item number. Manuscripts, uplicate, may be sent to any one of the four editors. Please classify according to the scheme of Math. Revieu Idex to Vol. 39. All other communications should be addressed to the managing editor, or Elaine Bart Iniversity of California, Los Angeles, California, 90024.

100 reprints are provided free for each article, only if page charges have been substantially pai dditional copies may be obtained at cost in multiples of 50 .

The Pacific Journal of Mathematics is issued monthly as of January 1966. Regular subscription rate: \$72. year (6 Vols., 12 issues). Special rate: $\$ 36.00$ a year to individual members of supporting institutions.

Subscriptions, orders for numbers issued in the last three calendar years, and changes of address should ent to Pacific Journal of Mathematics, 103 Highland Boulevard, Berkeley, California, 94708.

UBLISHED BY PACIFIC JOURNAL OF MATHEMATICS, A NON-PROFIT CORPORATION

Printed at Jerusalem Academic Press, POB 2390, Jerusalem, Israel.

Copyright (C) 1978 Pacific Journal of Mathematics

All Rights Reserved 


\section{Pacific Journal of Mathematics}

\section{Vol. 75, No. $1 \quad$ September, 1978}

Mieczyslaw Altman, General solvability theorems

Denise Amar and Eric Amar, Sur les suites d'interpolation en plusieurs variables ..........................................

Herbert Stanley Bear, Jr. and Gerald Norman Hile, Algebras which satisfy a second order linear partial differential equation ..................

Marilyn Breen, Sets in $R^{d}$ having $(d-2)$-dimensional kernels ............

Gavin Brown and William Moran, Analytic discs in the maximal ideal space

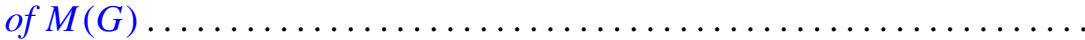

Ronald P. Brown, Quadratic forms with prescribed Stiefel-Whitney

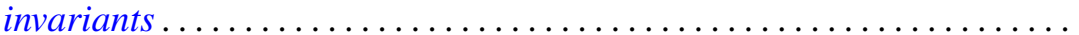

Gulbank D. Chakerian and H. Groemer, On coverings of Euclidean space by convex sets

S. Feigelstock and Z. Schlussel, Principal ideal and Noetherian groups.....

Ralph S. Freese and James Bryant Nation, Projective lattices ............

Harry Gingold, Uniqueness of linear boundary value problems for

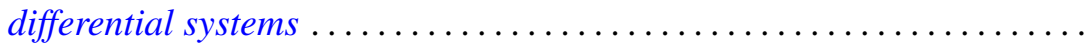

John R. Hedstrom and Evan Green Houston, Jr., Pseudo-valuation domains. . .

William Josephson, Coallocation between lattices with applications to measure extensions

M. Koskela, A characterization of non-negative matrix operators on $l^{p}$ to $l^{q}$ with $\infty>p \geq q>1$

Kurt Kreith and Charles Andrew Swanson, Conjugate points for nonlinear differential equations...........................

Shoji Kyuno, On prime gamma rings ........................ 185

Alois Andreas Lechicki, On bounded and subcontinuous multifunctions ..

Roberto Longo, A simple proof of the existence of modular automorphisms in approximately finite-dimensional von Neumann algebras ...

Kenneth Millett, Obstructions to pseudoisotopy implying isotopy for

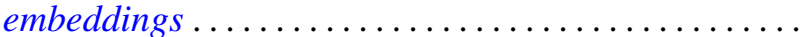

William F. Moss and John Piepenbrink, Positive solutions of elliptic equations. .

Mitsuru Nakai and Leo Sario, Duffin's function and Hadamard's

conjecture

Mohan S. Putcha, Word equations in some geometric semigroups ...

Walter Rudin, Peak-interpolation sets of class $C^{1} \ldots \ldots \ldots$

Elias Saab, On the Radon-Nikodým property in a class of locally convex

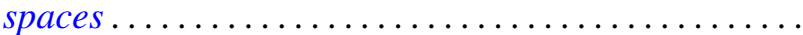

